9. Partridge M. Metered-dose inhalers and CFCs; what respiratory physicians need to know. Respir Med 1994; 88: 645-7.

10. Woodcock A. CFCs and inhalers. Lancet 1994; 344: 812. 11. Thompson P. Evaluating the therapeutic airways response to inhaled steroids. Clinical implication of improved targeting of inhaled steroids to the airways. European Respiratory Society Symposium: Stockholm 1996

12. Global Initiative for Asthma (GINA). Global strategy for asthma management and prevention. National Heart, Lung and Blood Institute (NHLBI)/World Health Organisation Workshop Report 1993. National Institutes of Health, NHLBI, Publication no. 95-3659. January 1995.

13. Cline A and Amies M. Patient preference for CFC-free aerosol metered dose inhaler (PMDI) replacement for current beclomethasone diproprionate (BDP) CFC PMDI. Eur Resp J 1996; 9(23): 254s

\title{
The role of inhaled steroids in COPD: the search for evidence
}

\author{
J Haughney
}

$\mathrm{C}$ hronic obstructive pulmonary disease (COPD) has for a long time been the 'Cinderella' respiratory condition in general practice. GPs and practice nurses see many patients with COPD, whose management is less clear than for asthma, with its evidence-based guidelines and treatment protocols. One of the most controversial questions in COPD is the value of inhaled corticosteroids. This is due to a fundamental lack of understanding about the underlying nature of the condition and the lack of data on long-term treatment.

COPD is defined as a chronic, slowly progressive disorder characterised by airways obstruction which does not change markedly over several months. Impairment of lung function is largely fixed but is partially reversible by bronchodilator (or other) therapy. It is a general term that covers many conditions which are now recognised as sharing the same underlying lung problems, including chronic bronchitis and emphysema. Many patients may also have some elements of asthma, explaining why lung function may be partially reversible. In general practice, COPD mainly affects older age groups and most cases are caused by tobacco smoking. Current indications suggest that the management of COPD may be suboptimal and greater efforts are warranted on the part of the GP.

COPD causes significantly more mortality and morbidity than other causes of airflow limitation in adults. In 1992, there were 26,033 deaths attributed to COPD, chronic bronchitis and emphysema in England and Wales, which accounts for 6.4 per cent of all male and 3.9 per cent of all female deaths. ${ }^{1}$ As one of the most common conditions seen in GP surgeries in patients over the age of 55, COPD has significant implications for a GPs workload. It results in two to four times more consultations than angina.

THE RATIONALE FOR USING CORTICOSTEROIDS IN COPD Many GPs already use inhaled corticosteroids in addition to inhaled bronchodilators in COPD, but is any real benefit achieved? There are at least two possible reasons why COPD patients might respond to antiinflammatory treatment, despite the general acknowledgment that the loss of lung tissue elasticity is relatively fixed. Some asthma patients may be misdiagnosed as COPD. The British Thoracic Society COPD guidelines ${ }^{1}$ point out that the differentiation of severe COPD from chronic severe asthma can be difficult because some degree of reversibility (shown as improvement in $\mathrm{FEV}_{1}$ ) can be achieved in the vast majority of patients. A spirometric response is considered positive if the $\mathrm{FEV}_{1}$ increases by $200 \mathrm{ml}$ and $15 \%$ from the baseline value. The pathological changes of bronchial asthma in the large airways can co-exist with those of COPD, which predominantly affects the small airways. Patients with COPD who respond to steroids may have a degree of inflammation which may be component specific to the disease.

While the presence of inflammatory changes in the airways of patients with COPD provides a rationale for the use of corticosteroids, ${ }^{2}$ however, the association between these changes, lung function and the therapeutic response to corticosteroids has not yet been clearly established. Whether a response to $30 \mathrm{mg}$ of prednisolone over two weeks predicts an ongoing improvement with inhaled corticosteroid treatment is debatable and results are awaited from ongoing studies.,4

\section{THE VALUE OF INHALED STEROIDS IN COPD}

Several uncontrolled retrospective studies suggest that long-term oral corticosteroids may slow the decline in $\mathrm{FEV}_{1}$ in patients with COPD ${ }^{5,6}$ However, long-term use of oral steroids would not generally be recommended, because of the risk of systemic side-effects. Inhaled corticosteroids offer an option for achieving similar benefits with less systemic side-effects.

An early trial suggested an improvement in $\mathrm{FEV}_{1}$ and reduction in the decline in $\mathrm{FEV}_{1}$ over one year's treatment with inhaled beclomethasone. ${ }^{7}$ A two year study with inhaled budesonide ( $1600 \mathrm{mcg} /$ day) showed significant reduction in respiratory symptoms, with a halving of the median decline in $\mathrm{FEV}_{1}$ in a group of nonallergic patients with COPD $(30 \mathrm{ml} / \mathrm{yr}$ in the budesonide group, compared to $60 \mathrm{ml} / \mathrm{yr}$ in the placebo group). The number of patients withdrawing due to pulmonary problems was significantly higher in the placebo group. ${ }^{8}$

\section{ONGOING TRIALS IN COPD}

What is needed to clarify the position is data from longterm studies investigating the use of inhaled corticosteroids in COPD. Three major multicentre trials are currently underway - The European Respiratory Society Study in

\author{
John Haughney \\ General Practitioner and \\ Editorial Board Member \\ Alison Lea Medical \\ Centre, Calderwood, East \\ Kilbride G74 3BE. \\ Date received: 08/12/97 \\ Date accepted: 12/01/98 \\ Asthma in Gen Pract 1998 \\ 6(1):5-7.
}


Chronic Obstructive Pulmonary Disease (EUROSCOP), ${ }^{4}$ the Copenhagen Lung Study ${ }^{9}$ and Inhaled Steroids in Obstructive Lung Disease in Europe (ISOLDE).

\section{EUROSCOP}

This was the first multicentre study to be carried out by the European Respiratory Society, involving 38 centres throughout Europe. It was based on the hypothesis that inflammation plays an important part in the pathogenesis of COPD and is investigating whether inhaled budesonide can slow the decline in $\mathrm{FEV}_{1}$ in smokers with the condition. More than 2000 patients were recruited into the study - all were $30-65$ years old and current smokers, smoking five or more cigarettes per day. Their $\mathrm{FEV}_{1} / \mathrm{FVC}$ ratios were less than 70 per cent and reversibility to bronchodilator ( $\beta$-agonist) was less than 10 per cent. Patients with a history of asthma, allergic rhinitis or allergic eczema were excluded in an effort to study 'pure' COPD.

The initial six month run-in period of the trial was based on efforts to achieve smoking cessation for three months, followed by a compliance check for three months. Results showed that only 12.7 per cent of patients gave up smoking - a depressingly low figure, but probably better than might be achieved outside trials. A total of 1277 patients were then randomised to budesonide ( $800 \mathrm{mcg}$ per day) or placebo, with 912 finishing the three year study. The results require careful interpretation, but there was a reduction in the decline in $\mathrm{FEV}_{1}$ with inhaled budesonide in some subgroups of the study population. Intriguingly, benefits seem to have been greater in women than in men.

\section{The Copenhagen Lung Study}

This study aims to assess the long-term efficacy of inhaled budesonide by measuring changes in $\mathrm{FEV}_{1}$ in patients with moderate COPD. It is part of the Copenhagen City Heart Study, ${ }^{10}$ which has been following up more than 14,000 people since the mid1970s. COPD is being studied in people aged 30-70 years, with $\mathrm{FEV}_{1} / \mathrm{FVC}$ ratios $<0.7$ (70 per cent) and little reversibility to $B$-agonists. They have been randomised to six-months' treatment with budesonide (1200 mcg daily) followed by a further six months (800 mcg daily) or placebo. Lung function was measured every two weeks at the start of treatment, at six months and at the end of the trial. A total of 1118 subjects were identified with obstructive lung disease. Of the 604 tested for reversibility, 290 were found to be suitable for inclusion. The average $\mathrm{FEV}_{1}$ was 79 per cent of predicted. A total of 135 patients have now

Box 1: British Thoracic Society Guidelines on COPD - treatment recommendations

Treatment recommendations
- MILD (FEV $160-80$ per cent predicted) - bronchodilator
(short acting $\mathrm{B}_{2}$-agonists or inhaled anticholinergics)
as required.
- MODERA TE (FEV $140-59$ per cent predicted) -
bronchodilator prn, but regular therapy may also be
needed with one drug or combination. Consider
corticosteroid trials in all patients.
- SEVERE (FEV $1<40$ per cent predicted) - combination
therapy regular $\mathrm{B}_{2}$-agonists plus anticholinergic; $^{- \text {aging }}$ consider steroid trial; assess for home nebuliser using
BTS guidelines; oxygen
- NON-DRUG MEASURES - smoking cessation,
exercise, vaccination against influenza.

\section{ISOLDE}

This is a UK-based, multicentre, double-blind, placebo-controlled study of fluticasone $(500 \mathrm{mcg}$ twice daily) in patients with moderate to severe COPD, with the main outcome measure being the rate of decline of post-bronchodilator $\mathrm{FEV}_{1}$ over three years. Patients recruited have a diagnosis of COPD, are aged 40-75 years and have post-bronchodilator $F V_{1}$ values less than 70 per cent of predicted. A total of 753 patients have been randomised, with 46 per cent being current smokers. The average prebronchodilator $\mathrm{FEV}_{1}$ was 1.24 litres, suggesting the group has severe COPD. The trial finished in March 1998 and the results are being presented at the European Respiratory Society this year.

These trials are trying to focus on patients with 'pure' COPD, which should provide a clear picture of whether inhaled steroids are helpful. It is important to remember that many patients seen in general practice may also have some element of asthma; spirometry assessment of reversibility is required in order to plan management for these patients.

\section{CURRENT USE OF INHALED STEROIDS IN COPD}

The BTS guidelines ${ }^{1}$ suggest that a trial of oral steroids (two weeks of $30 \mathrm{mg}$ prednisolone daily) is indicated in assessing moderate to severe COPD. This has achieved a significant spirometric response in 1020 per cent of patients with clinically stable COPD. ${ }^{11}$ The guidelines suggest that a positive response to corticosteroids justifies prescription of regular inhaled steroids. They recommend giving inhaled steroids (up to the equivalent of $1000 \mu \mathrm{g}$ beclomethasone, $800 \mu \mathrm{g}$ budesonide or $500 \mu \mathrm{g}$ fluticasone/day) to patients who show an objective response to corticosteroids, either oral or inhaled.

Until results are published from the major ongoing trials with inhaled corticosteroids in COPD, the best we can do is to make efforts to clearly differentiate the condition from asthma, ideally by using spirometry to investigate reversibility. Smoking cessation is the single most important management step in COPD. Treatment should be tailored to the individual patient, monitoring lung function and symptomatic response to inhaled therapy and targeting use of inhaled steroids to those patients who respond to a two week trial.

\section{References}

1. British Thoracic Society. Guidelines for the Management of Chronic Obstructive Pulmonary Disease. Thorax 1997; 52(5): S1-S32.

2. Saetta M, di Stefano A, Maestrelli P et al. Activated T lymphocytes and macrophages in bronchial mucosa of subjects with chronic bronchitis. Am Rev Respir Dis 1993; 147: 301-6. 3. Weir D C, Gove R I, Robertson A S et al. Corticosteroid trials in non-asthmatic airflow obstruction: comparison of oral prednisolone and inhaled betamethasone diproprionate. Thorax 1990; 45: 112-17.

4. European Respiratory Society, 1997, verbal communication. 5. Postma D S, Peters I, Steenhuis E J et al. Severe chronic airflow obstruction: can corticosteroids slow down progression? Eur J Respir Dis 1985; 67: 56-64.

6. Postma D S, Peters I, Steenhuis E J et al. Moderately severe chronic airflow obstruction. Can corticosteroids slow down progression? Eur Respir J 1988; 1: 22-6.

7. Dompeling E, van Schayck E P, Molema J et al. Inhaled beclomethasone improves the course of asthma and COPD Eur Respir J 1992; 5: 945-52. 
8. Renkema T E, Schouten J P, Koeter G H et al. Effects of long-term treatment with corticosteroids in COPD. Chest 1996; 109(5): 1156-62.

9. Prescott $\mathrm{E}$ et al. Gender differences in smoking effects on lung function and risk of hospitalisation for COPD: results from a Danish longitudinal study. Eur Respir J 1997; 10(4): 822-7.
10. Nardestgaard B G et al. A prospective cardiovascular population study used in genetic epidemiology. The Copenhagen City Heart Study. Scand J Clin Invest Suppl 1996; 226: 65-71. 11. Callahan C M, Cittus R S, Katz B P. Oral corticosteroid therapy for patients with stable chronic obstructive pulmonary disease: a meta-analysis. Ann Intern Med 1991; 114: 216-23.

\title{
Barriers to the implementation of guidelines in general practice
}

\author{
K Khunti and M K Lakhani
}

$\mathrm{I}$ $\mathrm{n}$ recent years, there has been increasing interest in the use of clinical guidelines as a method of getting evidence-based recommendations into practice. This is as a result of some obvious problems with current clinical practice which include variation in care, delays in implementing research findings, the use of ineffective or even harmful treatments and an interest in costeffectiveness. ${ }^{1}$ Potential advantages of clinical guidelines include a reduction in inappropriate variations in practice, ensuring more effective management of common clinical conditions, the provision of a rational basis for referral, identifying learning needs for continuing medical education and improved control of health care costs. ${ }^{2}$ There is now a plethora of clinical guidelines, however, the evidence for their effectiveness in UK primary care is patchy. There is evidence that guidelines which are properly developed and effectively disseminated and implemented can lead to change in clinical practice and improvements in patient outcomes. ${ }^{3}$ Dissemination is defined as the process which aims to influence the clinicians' awareness, attitudes, knowledge, and understanding of the guidelines. Implementation is defined as the process which encourages clinicians to change their practice. ${ }^{3}$ Many different strategies are available for dissemination and implementation. However, before we discuss these, it is necessary to consider the development of guidelines.

The aim of developing clinical guidelines is to produce explicit recommendations that are both scientifically valid and helpful to clinicians in their everyday practice. There is now a greater awareness of the best methods of developing valid clinical guidelines. ${ }^{4.5}$ Practice guidelines are considered to be valid if, when they are appropriately followed, they lead to change. ${ }^{5}$ Guidelines are more valid if they are developed using systematic reviews by national or regional guideline development groups and have explicit links between the recommendations and scientific evidence. ${ }^{5}$ In addition, guidelines are more likely to be successful if they are developed by a multidisciplinary group containing representatives of the key disciplines involved, disseminated through educational interventions and implemented via patient specific reminders during consultation.

There are two major problems when using clinical guidelines in primary care. Firstly there are now a large number of guidelines which are sent to GPs, often on the same topic. The GP may not be able to assess the quality of all the guidelines. Secondly getting health care professionals to use guidelines is a major problem. Modifying clinicians' behaviour to conform more closely to practice guidelines has proved to be a difficult task.

There are many reasons why guidelines fail to be implemented in practice. Efforts to implement guidelines may be hampered by ambiguity or lack of specificity in clinical recommendations, or inconsistency of the recommendations with existing practice patterns or policies of health care organisations. Even though general practitioners may be well informed about a clinical guideline (dissemination), they may not perform according to the recommendations contained in the guideline (implementation).

Recently there has been increasing importance attached to the most effective strategies for implementing guidelines. Strategies specific to individual patients are more likely to encourage the adoption of guidelines and bring about change in patient care. ${ }^{3}$ Any guideline implementation strategy should have an impact at four levels;

- increasing knowledge,

- changing attitudes,

- changing behaviour,

- changing outcomes.

Guideline implementation involves an interactive proces with multiple intervention points and strategies often fail because of the complexities of the implementation process. Therefore, before guidelines are implemented, the obstacles to change should be identified. Robertson and colleagues have described a framework for integrating obstacles and strategies for change at personal level, at group level and at organisational level. ${ }^{7}$ Examples of the theories of obstacles to change at the doctor level include:

- Self-efficacy: GP who thinks that he or she lacks knowledge or ability.

- Willingness to change: GP who considers that current practice is adequate and is unwilling to consider change.

- Social influence: GP who considers that the source of the guideline is not reputable.

- Bereavement reaction: GP who fails to implement a guideline because he or she rejects or denies evidence of inadequate performance.

Grol has also produced a classification of change strategies for implementing guidelines which takes into account the different obstacles to change that might be relevant to doctors who are 'early adopters',

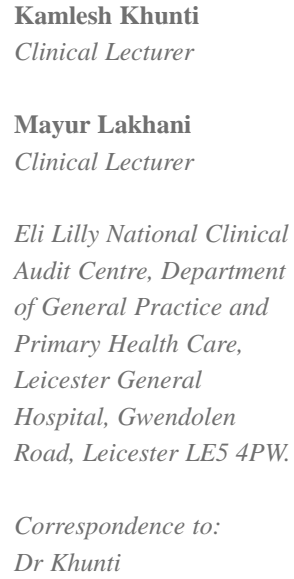

